\title{
Erratum to: Self-perceived uselessness and associated factors among older adults in China
}

\author{
Yuan Zhao', Jessica M. Sautter ${ }^{2}$, Li Qiu ${ }^{3}$ and Danan Gu+
}

\section{Erratum}

After the publication of this work [1] five corrections were discovered in the text. The corrections are as follows:

1. In the first line of the fourth paragraph in the section, Background, the text "non-Western cultures" should be "Western cultures".

2. In the first line of the section, Factors associated with self-perceived uselessness, the text "six sets" should be "five sets".

3. In Row 2 of Table 1, the text "Giving money/food to children-yes" should be "Giving money/food to children-no". But the figures in the same line should stay. The "no" category should be corresponding to 77.0.

4. In Row 3 of Table 1, the text "Giving money/food to children-no" should be "Giving money/food to children-yes". The figures in the same line should stay. The "yes" category should be corresponding to 23.0.

5. The last sentence of the first paragraph in section, Risk of self-perceived uselessness was lower in supportive and culturally traditional social environments, "The findings in Model III in Table 3 are similar to those in Table 2 except that some of these variables were still significant in Table 3" should be "The findings in Model III in Table 3 indicate that the association between social environments and self-perceived uselessness was weakened when the moderate frequency of self-perceived uselessness was compared to the low frequency".

\section{Author details}

'Ginling College and School of Geography Science, Nanjing Normal University, Nanjing, China. ${ }^{2}$ Department of Behavioral and Social Sciences, University of the Sciences, Philadelphia, PA, USA. ${ }^{3}$ Independent Researcher,
New York, NY, USA. ${ }^{4}$ United Nations Population Division, Two UN Plaza, DC2-1910, New York, NY, USA.

Received: 26 April 2017 Accepted: 26 April 2017

Published online: 02 May 2017

Reference

1. Zhao Y, Sautter JM, Qiu L, Gu D. Self-perceived uselessness and associated factors among older adults in China. BMC Geriatr. 2017;17:12.

* Correspondence: gudanan@yahoo.com

${ }^{4}$ United Nations Population Division, Two UN Plaza, DC2-1910, New York, NY, USA

Full list of author information is available at the end of the article 